\title{
Error Bounds in the Pointwise Approximation of Solutions of Elastic Plate Problems
}

\author{
J. H. Bramble* and L. E. Payne*
}

(May 27, 1963)

\begin{abstract}
Methods are presented for obtaining upper and lower pointwise bounds for the deflection (and its derivatives) in certain physically interesting elastic plate problems.
\end{abstract}

\section{Introduction}

In a recent paper $[1]^{1}$ the authors presented a new method for obtaining pointwise bounds in the first boundary value problem for elastic plates. The method is based on a mean value inequality and an a priori inequality for biharmonic functions. In this paper we use the same techniques to obtain pointwise bounds in terms of known data for the deflection and its derivatives in the second boundary value problem for an elastic plate, i.e., the displacement and bending moment prescribed on the boundary of the plate. In addition we derive pointwise bounds for the solution (and its derivatives) of the third boundary value problem, i.e., the bending moment and reaction prescribed on the plate boundary. In each of these problems bounds at an interior point for the deflection and any derivative up to and including the third are obtained in a straightforward manner. The inequalities obtained, bound an arbitrary (sufficiently smooth) function at a point in a region in terms of the data of the given problem. Thus if one applies the inequality to the difference between a desired solution (with known data) and an arbitrary trial function, it yields a bound for the error in the approximation of the unknown solution by the known trial function. The trial functions need only possess piecewise continuous fourth derivatives and are not required to satisfy any differential equation or boundary conditions. If the applied loading is sufficiently smooth in a neighborhood of the point at which bounds are sought, it is possible to obtain in addition to the above mentioned results, bounds for higher derivatives of the deflection in either of the two problems considered here. We note the somewhat unexpected fact that it is considerably easier to obtain bounds in the third boundary value problem than in either of the first two.

- In a subsequent paper the authors wil present methods for obtaining pointwise bounds in mixed boundary value problems for elastic plates.

\section{Notation and Definitions}

Throughout this paper we assume the plate to occupy a two dimensional bounded region $R$ with boundary $C$. If $R$ is not polygonal it is necessary (for our method) in the second boundary value problem to assume that $C$ have bounded curvature. We make use of the summation convention throughout this paper and employ a comma to denote differentiation, e.g., $W,{ }_{i}=\partial W / \partial x^{i}$. The symbol $\sigma$ is used to denote Poisson's ratio and $D$ to signify the plate rigidity. The symbol $\Delta$ denotes the Laplace operator and $\Delta^{2}$ the biharmonic operator. Additional notation will be defined as need arises in the text.

*University of Maryland, College Park, Md. Part time at National Bureau of Standards, Washington, D.C.

${ }^{1}$ Figures in brackets indicate the literature references at the end of this paper. 


\section{Second Boundary Value Problem for Elastic Plates}

The boundary value problem considered in this section may be stated as:

in $R$ and

$$
\Delta^{2} u=f
$$

$$
u=g, M(u)=h
$$

on $C$. The quantities $f, g, h$ are prescribed data and $M(u)$ is the bending moment; i.e.,

$$
M(u)=-D\left[\Delta u-(1-\sigma)\left(\frac{\partial^{2} u}{\partial s^{2}}+\frac{1}{\rho} \frac{\partial u}{\partial n}\right)\right] .
$$

In (3.3) $\frac{\partial^{2} u}{\partial s^{2}}$ denotes the second tangential derivative of $u, \partial u / \partial n$ the normal derivative and $\rho$ is the radius of curvature on $C$.

It is possible to obtain bounds for the strain energy, but since this quantity is not usually of physical interest we do not present a bound for it here. We indicate now a method for obtaining pointwise bounds for $u$ and its derivatives. The result may be stated as follows:

THEOREM I: For any biharmonic function $\mathrm{V}$ with piecewise continuous fourth derivatives in a domain $\mathrm{R}$ whose boundary $\mathrm{C}$ has bounded curvature, the following a priori bound holds for any $\mathrm{p}$ th order derivative of $\mathrm{V}$ at a point $\mathrm{O}$ in $\mathrm{R}$ :

$$
\left|V^{(p)}(O)\right|^{2} \leqslant K_{1}(p) \oint_{C} V^{2} d s+K_{2}(p) \oint_{C}\left(\frac{\partial V}{\partial s}\right)^{2} d s+K_{3}(p) \oint_{C}\left(\frac{\partial^{2} V}{\partial s^{2}}\right)^{2} d s+K_{4}(p) \oint_{C}[M(V)]^{2} d s,
$$

where the constants $K_{1}(p), \ldots ., K_{4}(p)$ are explicitly determined.

Before proving this theorem we indicate how such an a priori inequality leads to pointwise bounds for $u$ and its derivatives in $R$. If we denote by $\varphi$ any particular solution of (3.1) and set $V=u-\varphi-\bar{B}$, where $\vec{B}$ is biharmonic, then by choosing the data of $\bar{B}$ to approximate that of $u-\varphi$ (the usual Rayleigh-Ritz technique ${ }^{2}$ ) we obtain pointwise bounds for $u$ and its derivatives.

In proving theorem I we make use of mean value inequalities derived in [1], i.e., if $S_{r}$ denotes the interior of a sphere contained in $R$ and of radius $r$ about $O$, then

$$
\begin{aligned}
|V(O)|^{2} & \leqslant \frac{4}{\pi r^{2}} \int_{S_{r}} V^{2} d x d y \leqslant \frac{4}{\pi r^{2}} \int_{R} V^{2} d x d y \\
\left|V^{(1)}(O)\right|^{2} & \leqslant \frac{36}{\pi r^{4}} \int_{S_{r}} V^{2} d x d y \leqslant \frac{36}{\pi r^{4}} \int_{R} V^{2} d x d y
\end{aligned}
$$

with similar expressions for the higher derivatives (see [1]). Thus in order to establish (3.4) it is sufficient to obtain a bound $\int_{R} V^{2} d x d y$ in terms of the quantities on the right of (3.4).

To this end we decompose $V$ as

$$
V=B+H
$$

where

$$
\Delta H=O
$$

in $R$ and

$$
H=V
$$

on $C$. Then

$$
\Delta^{2} B=O
$$

in $R$ and

$$
\begin{gathered}
B=O \\
M(B)=M(V)-M(H)
\end{gathered}
$$


on $C$. Clearly

$$
\int_{R} V^{2} d x d y \leqslant 2\left\{\int_{R} B^{2} d x d y+\int_{R} H^{2} d x d y\right\}
$$

We first derive a bound for $\int_{R} B^{2} d x d y$. To do this we introduce an auxiliary function $W$ satisfying

$$
\Delta^{2} W=B
$$

in $R$ and

$$
W=O, M(W)=O
$$

on $C$. Then using the divergence theorem we obtain

$$
\int_{R} B^{2} d x d y=\int_{R} B \Delta^{2} W d x d y=-\frac{1}{D} \oint_{C} M(B) \frac{\partial W}{\partial n} d s .
$$

Thus by Schwarz's inequality

$$
\int_{R} B^{2} d x d y \leqslant\left[\frac{1}{D^{2}} \oint_{C}[M(B)]^{2} t^{-1} d s \oint_{C} t\left(\frac{\partial W}{\partial n}\right)^{2} d s\right]^{1 / 2},
$$

where $t$ is any positive function on $C$.

In order to bound the last integral in (3.16) we introduce a vector field with components $f^{i}$ such that $f^{i} n_{i}>O$ on $C$. Then setting

$$
t=f^{i} n_{i}
$$

we have

$$
2 \int_{R} f^{i} W,{ }_{i} \Delta W d x d y=\oint_{C} t\left(\frac{\partial W}{\partial n}\right)^{2} d s+\int_{R}\left(f,{ }_{i} \delta_{i}^{\prime}-2 f,{ }_{i}\right) W,{ }_{i} W,{ }_{l} d x d y .
$$

This is one form of an identity due to Rellich (cf. [5]).

If the derivatives of $f^{i}$ are bounded in $R$, we obtain by applying the arithmetic-geometric mean inequality to (3.18)

$$
\oint_{C} t\left(\frac{\partial W}{\partial n}\right)^{2} d s \leqslant\left(c+\frac{b}{\alpha}\right) \int_{R} W,{ }_{i} W,{ }_{i} d x d y+\alpha \int_{R}(\Delta W)^{2} d x d y .
$$

Here $c$ is just some bound for the largest eigenvalue of the matrix $2 f_{,},{ }_{i}-f_{,}^{k}{ }_{k} \delta_{i}^{l}$, i.e.,

$$
\int_{R}\left(2 f,{ }_{i}-f_{,}^{k}{ }_{k} \delta_{i}^{l}\right) W \cdot{ }_{i} W,{ }_{l} d x d y \leqslant c \int_{R} W,{ }_{i} W,{ }_{i} d x d y,
$$

and

$$
b=\operatorname{Max}\left(f^{i} f^{i}\right) .
$$

In (3.19), $\alpha$ is any positive constant. For a star shaped boundary we may choose $f^{i}=x^{i}$ in which case we may take $c=0$. Now, since $W$ vanishes on $C$, it is clear that

$$
\int_{R} W^{2} d x d y \leqslant \frac{1}{\lambda_{1}} \int_{R} W,{ }_{i} W,{ }_{i} d x d y \leqslant \frac{1}{\lambda_{1}^{2}} \int_{R}(\Delta W)^{2} d x d y
$$

where $\lambda_{1}$ is the first eigenvalue of the fixed membrane problem for $R .^{3}$

The strain energy $E(W, W)$ is given by

$$
E(W, W)=\frac{D}{2}\left\{\int_{R}\left[\sigma(\Delta W)^{2} d x d y+(1-\sigma) W,{ }_{i j} W,{ }_{i j}\right] d x d y\right\} .
$$

\footnotetext{
${ }^{3}$ For a discussion of the fixed membrane problem see for instance Courant, R., and Hilbert, D., Methods of Mathematical Physics 1, Interscience (1953).
} 
It follows easily, since $W,{ }_{i j} W,{ }_{i j} \geqslant \frac{1}{2}(\Delta W)^{2}$, that

$$
\int_{R}(\Delta W)^{2} d x d y \leqslant \frac{2}{1+\sigma}\left[\frac{2}{D} E(W, W)\right]
$$

and, since $W$ and $M(W)$ vanish on $C, \frac{2}{D} E(W, W)=\int_{R} W \Delta^{2} W d x d y$. Hence

$$
\int_{R}(\Delta W)^{2} d x d y \leqslant \frac{2}{(1+\sigma)} \int_{R} W \Delta^{2} W d x d y .
$$

From (3.22)

$$
\int_{R} W^{2} d x d y \leqslant \frac{1}{\lambda_{1}^{2}} \int_{R}(\Delta W)^{2} d x d y
$$

We thus obtain, using Schwarz's inequality on (3.25) and inserting (3.26),

$$
\int_{R}(\Delta W)^{2} d x d y \leqslant\left[\frac{2}{(1+\sigma) \lambda_{1}}\right]^{2} \int_{R}\left(\Delta^{2} W\right)^{2} d x d y .
$$

Combining (3.19), (3.22) and (3.26) we now have (choosing $\left.\alpha=\left[b / \lambda_{1}\right]^{1 / 2}\right)$,

$$
\oint_{C} t\left(\frac{\partial W}{\partial n}\right)^{2} d s \leqslant \frac{4}{(1+\sigma)^{2} \lambda_{1}^{3}}\left(c+2\left[b \lambda_{1}\right]^{1 / 2}\right) \int_{R}\left(\Delta^{2} W\right)^{2} d x d y
$$

In (3.28) we may use any lower bound for $\lambda_{1}$, e.g., that obtained from the isoperimetric inequality of Faber [3] and Krahn [4]

$$
\lambda_{1} \leqslant \pi j_{0}^{2} / A
$$

Here $j_{0}$ is the first zero of the Bessel function $J_{0}$ and $A$ denotes the area of $R$. We now set

$$
A_{1}=\frac{4}{(1+\sigma)^{2} \bar{\lambda}_{1}^{3}}\left(c+2\left\lceil b \bar{\lambda}_{1}\right\rceil^{1 / 2}\right)
$$

where $\bar{\lambda}_{1}$ is any lower bound for $\lambda_{1}$. Then from (3.16) we obtain (recalling (3.13))

$$
\int_{R} B^{2} d x d y \leqslant \frac{A_{1}}{D^{2}} \oint_{C}[M(B)]^{2} d s
$$

From (3.3), (3.9), and (3.11) we have

$$
M(B)=M(V)-D(1-\sigma)\left[\frac{\partial^{2} V}{\partial s^{2}}+\frac{1}{\rho} \frac{\partial H}{\partial n}\right] .
$$

Thus

$$
\int_{R} B^{2} d x d y \leqslant \frac{2 A_{1}}{D^{2}}\left\{\oint_{C}\left[M(V)-D(1-\sigma) \frac{\partial^{2} V}{\partial s^{2}}\right]^{2} d s+D^{2}(1-\sigma)^{2} \oint_{C} \frac{1}{\rho^{2}}\left(\frac{\partial H}{\partial n}\right)^{2} d s\right\} .
$$

We assume that the radius of curvature is bounded away from zero on $C$, i.e.,

$$
\rho^{2} \geqslant \beta>0
$$

on $C$.

In $[2,5]$ the inequality

$$
\begin{aligned}
t_{m} \oint_{C}\left(\frac{\partial H}{\partial n}\right)^{2} d s \leqslant \oint_{C} t^{-1}\left[2 f^{i} \frac{\partial H}{\partial s} \frac{\partial x^{i}}{\partial s}-c H\right]^{2} d s & +2 \oint_{C} t\left(\frac{\partial H}{\partial s}\right)^{2} d s \\
& \leqslant \oint_{C} t^{-1}\left[2 f^{i} \frac{\partial V}{\partial s} \frac{\partial x^{i}}{\partial s}-c V\right]^{2} d s+2 \oint_{C} t\left(\frac{\partial V}{\partial s}\right)^{2} d s
\end{aligned}
$$

was derived. Here $t_{m}$ denotes the minimum value of $t$ on $C$, and $c$ is given by (3.20). Inserting 
(3.34) into (3.32) we then obtain (using 3.33) the inequality

$$
\begin{aligned}
\int_{R} B^{2} d x d y \leqslant 2 A_{1}\left\{D^{-2} \oint_{C}[M(V)-D\right. & \left.(1-\sigma) \frac{\partial^{2} V}{\partial s^{2}}\right]^{2} d s \\
& \left.+\frac{(1-\sigma)^{2}}{\beta t_{m}}\left[\oint_{C} t^{-1}\left[2 f^{i} \frac{\partial x^{i}}{\partial s} \frac{\partial V}{\partial s}-c V\right]^{2} d s+2 \oint_{C} t\left(\frac{\partial V}{\partial s}\right)^{2} d s\right]\right\} .
\end{aligned}
$$

Again, for a star-shaped boundary, we may take $f^{i}=x^{i}\left(x^{1}=x, x^{2}=y\right)$ and $c=0$, since $x^{i} n_{i}>0$ is just the condition for star-shapedness.

It remains to determine a bound for $\int_{R} H^{2} d x d y$ in (3.12). Such a bound, however, was given in [1]; i.e.,

$$
\int_{R} H^{2} d x d y \leqslant k_{2} \oint_{C} H^{2} d s=k_{2} \oint_{C} V^{2} d s
$$

where $k_{2}$ is given by

$$
k_{2}=\frac{1}{\lambda_{1}}\left[4 \lambda_{1}\left(f^{i} f^{i}\right)_{M}+c^{2}+2 c \inf \left\{\left|f_{, i}^{i}\right|_{M} 2 \lambda_{1}\left[f^{i} f^{i}\right]_{M}{ }^{1 / 2}\right\}\right]_{1 / 2} .
$$

The symbol ( $)_{M}$ denotes the maximum value of the quantity in parentheses.

Combining (3.35) and (3.36) and inserting into (3.12) we obtain the inequality

$$
\begin{array}{r}
\int_{R} V^{2} d x d y \leqslant 2 A_{1}\left\{2 D^{-2} \oint_{C}\left[M(V)-D(1-\sigma) \frac{\partial^{2} V}{\partial s^{2}}\right]^{2} d s+\frac{2(1-\sigma)^{2}}{\beta t_{m}}\left[\oint_{C} t^{-1}\left[f^{i} \frac{\partial x^{i}}{\partial s} \frac{\partial V}{\partial s}-C V\right]^{2} d s\right.\right. \\
\left.\left.+\oint_{C} t\left(\frac{\partial V}{\partial s}\right)^{2} d s\right]+\frac{k_{2}}{A_{1}} \oint_{C} V^{2} d s\right\} .
\end{array}
$$

The insertion of (3.38) into (3.5), (3.6) or the similar expressions for higher derivatives yields an expression which is not precisely in the form (3.4). However, (3.4) can be easily obtained if the arithmetic-geometric mean inequality is used in (3.38). This completes the proof of theorem I.

In some cases it may be difficult to compute a particular solution $\varphi$ and its derivatives in $R$ and on $C$. In that case we may approximate $u$ by any sufficiently smooth function $\psi$ and decompose as follows:

where

$$
u-\psi=V+W
$$

in $R$ and

$$
\Delta^{2} V=O
$$

on $C$, while

$$
V=u-\psi, M(V)=M(u-\psi)
$$

in $R$ and

$$
\Delta^{2} W=\Delta^{2}(u-\psi)
$$

on $C$.

$$
W=O, M(W)=O
$$
Clearly,

We have already established a bound (3.4) for $V^{(p)}(O)$. We seek now a bound for $W^{(p)}(O)$.

$$
W(P)=\int_{R} G\left(P, P^{\prime}\right) \Delta^{2}(u-\psi) d x d y
$$

where $G\left(P, P^{\prime}\right)$ is the Green's function in the simply supported plate problem, i.e., $G\left(P, P^{\prime}\right)$ denotes the deflection of a simply supported plate at a point $P^{\prime}$ in $R$ due to a unit point load at $P{ }^{4}$ We now approximate $W(P)$ by

$$
\bar{W}(P)=\int_{R}\left[\Gamma\left(P, P^{\prime}\right)+\bar{B}\right] \Delta^{2}(u-\psi) d x d y,
$$

${ }^{4}$ For a discussion of $G$ ef. S. Timoshenko, Theory of plates and shells, pp. 156-167 (McGraw-Hill, 1940). 
where

$$
\Gamma\left(P, P^{\prime}\right)=\frac{1}{8 \pi} r_{P P^{\prime}}^{2} \log \frac{1}{r_{P P^{\prime}}},
$$

the singular part of $G\left(P, P^{\prime}\right)$, and $\bar{B}$ denotes any biharmonic function (possibly zero). We approximate a $p$ th derivative, $W^{(p)}(P)$, by $\bar{W}^{p}(P)$, where

$$
\bar{W}^{p}(P)=\left[\int_{R} \Gamma\left(P, P^{\prime}\right) \Delta^{2}(u-\psi) d x d y\right]^{(p)}+\int_{R} \bar{B}^{p} \Delta^{2}(u-\psi) d x d y .
$$

Here [ $]^{(p)}$ denotes the $p$ th derivative with respect to the variables of the point $P$, and $\bar{B}^{p}$ is an arbitrary biharmonic function, which may be taken identically zero. Clearly then at an interior point $P$

$$
W^{(p)}(P)-\bar{W}^{p}(P)=\int_{R}\left[G\left(P, P^{\prime}\right)-\Gamma\left(P, P^{\prime}\right)\right]^{(p)} \Delta^{2}(u-\psi) d x d y-\int_{R} \bar{B}^{p} \Delta^{2}(u-\psi) d x d y .
$$

The order of differentiation and integration is interchangeable in the first integral since $G\left(P, P^{\prime}\right)-$ $\Gamma\left(P, P^{\prime}\right)$ is biharmonic in $R$. For simplicity we adopt the convention that for a function of the two points $P$ and $P^{\prime}$, e.g., $\Gamma\left(P, P^{\prime}\right)$, the symbol $\Gamma^{(p)}\left(P, P^{\prime}\right)$ is to denote differentiation with respect to the variables of the first argument. Thus $\Gamma^{(p)}\left(P, P^{\prime}\right)$ denotes differentiation with respect to the variables of the point $P$ while $\Gamma^{(p)}\left(P^{\prime}, P\right)$ denotes differentiation with respect to the variables of the point $P^{\prime}$.

In view of (3.39) we obtain

$$
\left|u^{(p)}(O)-\psi^{(p)}(O)-\bar{W}^{p}(O)\right| \leqslant\left|V^{(p)}(O)\right|+\left|W^{(p)}(O)-\bar{W}^{p}(O)\right| .
$$

The term $\bar{W}^{p}(O)$ involves only known quantities and hence may be computed directly. If $p \leqslant 3$ the differentiation indicated in the first term on the right of (3.47) may be taken under the integral sign and placed on $\Gamma\left(P, P^{\prime}\right)$. The resulting integral exists provided $\Delta^{2}(u-\psi)=O\left(r_{P P^{\prime}}^{\epsilon}\right)$ as $P \rightarrow P^{\prime}$ for some positive $\epsilon$. If we wish to compute $\bar{W}^{p}(O)$ for $p \geqslant 3$ we decompose $\bar{W}^{p}(P)$ into

where

$$
\bar{W}^{p}(P)=\bar{W}_{1}^{p}(P)+\bar{W}_{2}^{p}(P)
$$

and

$$
\bar{W}_{1}^{p}(P)=\left[\int_{R} \Gamma\left(P, P^{\prime}\right) \Delta^{2}(u-\psi) d x d y\right]^{(p)}
$$

$$
\bar{W} p(P)=\int_{R} \bar{B}^{p} \Delta^{2}(u-\psi) d x d y
$$

Since $\bar{B}^{p}$ is biharmonic the last term presents no difficulty. In order to evaluate $\bar{W}_{1}^{p}(P)$ it is necessary to make some assumptions on the smoothness of $\Delta^{2}(u-\psi)$. We assume for the moment that $\Delta^{2}(u-\psi)$ has a sufficient number of derivatives in some spherical neighborhood $S_{\delta}$ of radius $\delta$ about the origin and that $P$ lies on the interior of $S_{\delta}$. Then

$$
\begin{aligned}
\bar{W}_{1}^{1}(P)=\int_{R} \Gamma^{(1)}\left(P, P^{\prime}\right) \Delta^{2}(u & -\psi) d x d y \\
& =\int_{R-S_{\delta}} \Gamma^{(1)}\left(P, P^{\prime}\right) \Delta^{2}(u-\psi) d x d y-\int_{S_{\delta}} \Gamma^{(1)}\left(P^{\prime}, P\right) \Delta^{2}(u-\psi) d x d y .
\end{aligned}
$$

We now integrate by parts in $S_{\delta}$ and obtain

$$
\begin{aligned}
\bar{W}_{1}^{1}(P)=\int_{R-S_{\delta}} \Gamma^{(1)}\left(P, P^{\prime}\right) \Delta^{2}(u-\psi) d x d y & \\
& \quad-\int_{\Sigma_{\delta}} n^{\prime} \Gamma\left(P, P^{\prime}\right) \Delta^{2}(u-\psi) d s+\int_{S_{\delta}} \Gamma\left(P, P^{\prime}\right)\left[\Delta^{2}(u-\psi)\right]^{(1)} d x d y .
\end{aligned}
$$


Here we have used the fact that $\Gamma\left(P, P^{\prime}\right)=\Gamma\left(P^{\prime}, P\right)$. In (3.54) $\Sigma_{\delta}$ denotes the boundary of $S_{\delta}$ and $n^{\prime}$ the component of the unit normal in the direction in which the differentiation is taken. Since $P$ lies interior to $S_{\delta}$ any derivatives of $\Gamma\left(P, P^{\prime}\right)$ with respect to the variables of the point $P$ are bounded on $\Sigma_{\delta}$ and in $R-S_{\delta}$. The last integral on the right of (3.54) may be differentiated 3 times with respect to the variables of $P$ and the order of integration and differentiation interchanged provided $\left[\Delta^{2}(u-\psi)\right]^{(1)}=O\left(r_{P P^{\prime}}^{\epsilon}{ }^{1}\right)$ for some positive $\epsilon$ as $P^{\prime} \rightarrow P$ in $S_{\delta}$. Letting $P \rightarrow O$ we thus obtain the value of $\bar{W}^{4}(O)$.

To define $\bar{W}^{p}(O)$ for $p>4$ we differentiate (3.54), make use of the fact that $\Gamma^{(1)}\left(P, P^{\prime}\right)=-$ $\Gamma^{(1)}\left(P^{\prime}, P\right)$, integrate the resulting final term by parts, and continue in this manner until we obtain an integral over $S_{\delta}$ which has the desired number of derivatives. We see in this way that for $\bar{W}^{p}(O)$ to remain finite, it is sufficient that $\Delta^{2}(u-\psi)$ have $p-4$ derivatives in $S_{\delta}$ and that the derivatives of order $p-3$ be $O\left(R_{P P^{\prime}}{ }^{1}\right)$ for some $\epsilon>O$ as $P^{\prime} \rightarrow P$ in $S_{\delta}$.

We need now only a bound for the last expression on the right of (3.49). To this end we use Schwarz's inequality in (3.48) to obtain

$$
\left[W^{(p)}(O)-\bar{W}^{p}(O)\right]^{2} \leqslant \int_{R}\left\{\left[G\left(O, P^{\prime}\right)-\Gamma\left(O, P^{\prime}\right)\right]^{(p)}-\bar{B}^{p}\right\} d x d y \int_{R}\left[\Delta^{2}(u-\psi)\right]^{2} d x d y .
$$

Since the differentiations in (3.55) are with respect to the variable of $P$ (before evaluation at $O$ ) the quantity $\left.\left\{G\left(O, P^{\prime}\right)-\Gamma\left(O, P^{\prime}\right)\right]^{(p)}-B^{p}\right\}$ is just another function $V$, i.e., $\Delta^{2} V=O$ in $R$, with $V$ and $M(V)$ given on $C$. Thus (3.38) is valid with $V$ replaced by $\left\{\left[G\left(O, P^{\prime}\right)-\Gamma\left(O, P^{\prime}\right)\right]^{(p)}-\bar{B}^{p}\right\}$ throughout. Since $G^{(p)}\left(O, P^{\prime}\right)$ and $M\left(G^{(p)}\left(O, P^{\prime}\right)\right)$ vanish on $C$ only the quantity $\left[\Gamma^{(p)}\left(O, P^{\prime}\right)+\bar{B}^{p}\right]$ appears for $V$ on the right. We note that the right hand side of (3.55) may be made small by choosing $\bar{B}^{p}$ to approximate the data of $-\Gamma^{(p)}\left(O, P^{\prime}\right)$ and/or by choosing $\psi$ to approximate the data of $u$.

Let us denote the right hand side of (3.38) by $I(V)$ and designate the constant in the mean value inequality for $\left|V^{(p)}(O)\right|$ as $C_{p}$, i.e.,

$$
\left|V^{(p)}(O)\right| \leqslant C_{p} \int_{R} V^{2} d x d y .
$$

Then our bound may be expressed in the simple form

$$
\left|u^{(p)}(O)-\psi^{(p)}(O)-\bar{W}^{(p)}(O)\right| \leqslant C_{p} I(u-\psi)+I\left(\Gamma^{(p)}\left(O, P^{\prime}\right)+\bar{B}^{p}\right) \int_{R}\left[\Delta^{2}(u-\psi)\right]^{2} d x d y .
$$

\section{Third Boundary Value Problem for Elastic Plates}

In this section we determine a priori bounds for a function $V$, assuming $\Delta^{2} V$ (the loading) is prescribed throughout a simply connected region $R$ and that the boundary values of $\bar{M}(V)$ and $\bar{Q}(V)$ are prescribed on $C$. Here $\bar{M}(V)$ is equal to $-M(V) / D$ where $D$ is given by $(2.1)$ and $M(V)$ is given by (3.3). Also

$$
\bar{Q}(V)=\frac{\partial}{\partial n}(\Delta V)-(1-\sigma)\left[\frac{\partial^{3} V}{\partial s^{2} \partial n}-\frac{\partial}{\partial s}\left(\frac{1}{\rho} \frac{\partial V}{\partial s}\right)\right] .
$$

The quantities $\bar{M}(V)$ and $\bar{Q}(V)$, are proportional to the bending moment and the reaction. We prove in this section the following theorem.

THEOREM II. Let $\mathrm{V}$ be any function with piecewise continuous fourth derivatives in $\mathrm{R}$ which satisfies the conditions

$$
\oint_{C} V d s=\oint_{C} \frac{\partial V}{\partial x} d s=\oint_{C} \frac{\partial V}{\partial y} d s=O
$$


Then at any point $\mathrm{O}$ in $R$,

$$
\left|V^{(p)}(O)\right|^{2} \leqslant k_{1} \oint_{C}[\bar{M}(V)]^{2} d s+k_{2} \oint_{C}[\bar{Q}(V)]^{2} d s+k_{3} \int_{R} \eta\left(\Delta^{2} V\right)^{2} d x d y
$$

where the constants $\mathrm{k}_{i}$ are explicitly determined, $\mathrm{p} \leqslant 3$, and $\eta$ is a known nonnegative function in $\mathrm{R}$.

Here, as before, $V^{(p)}(x, y)$ denotes any $p$ th order derivative of $V$. As indicated in the previous section once an a priori inequality of the type (4.2) has been derived it may be used to obtain pointwise bounds in the indicated boundary value problem.

Before proceeding further we define quantities $p_{2}$ and $q_{2}$ in terms of which the constants in (4.2) will be expressed. These quantities are defined as follows:

$$
p_{2}=\min _{\oint_{c} \chi d s=0} \frac{\int_{R} \chi,{ }_{i} \chi,{ }_{i} d x d y}{\oint_{C} \chi^{2} d s}
$$

where the minimum is taken over all piecewise continuously differentiable functions $\chi$ which satisfy the condition $\oint_{C} \chi d s=O$;

$$
q_{2}=\min _{\oint_{c} \psi d s=o} \frac{\oint_{C}\left(\frac{\partial \psi}{\partial s}\right)^{2} d s}{\oint_{C} \psi^{2} d s}
$$

where the minimum is taken among all functions $\psi$ continuously differentiable on $C$ and satisfying $\oint_{C} \psi d s=O$. The eigenvalues corresponding to the Rayleigh quotient in (4.3) are commonly referred to as Stekloff eigenvalues. They are the eigenvalues of the following problem

in $R$ and

$$
\Delta h=O
$$

$$
\frac{\partial h}{\partial n}-p h=O
$$

on $C$. In general, $p_{2}$ will not be known explicitly. However, for our purposes any positive lower bound $\bar{p}_{2}$ for $p_{2}$ will suffice. We indicate later how this lower bound is obtained. The eigenvalues corresponding to the Rayleigh quotient of (4.4) are the Wirtinger eigenvalues. They satisfy

$$
\frac{\partial^{2} u}{\partial s^{2}}+q u=O
$$

on $C$ and the proper continuity condition. The eq (4.7) is of course the equation of the vibrating string of length equal to the perimeter $L$ of $C$. Thus

$$
q_{2}=\frac{4 \pi^{2}}{L^{2}}
$$

We proceed now to the proof of theorem II. Let us again denote by $\Gamma\left(P, P^{\prime}\right)$ the fundamental solution of the biharmonic equation.

$$
\Gamma\left(P, P^{\prime}\right)=\frac{1}{8 \pi} r_{P P^{\prime}}^{2} \log \left(\frac{1}{r_{P P^{\prime}}}\right) .
$$


(We could add any biharmonic function $B$ to $\Gamma$ without altering the following results.) In terms of $\Gamma\left(P, P^{\prime}\right)$ we have

$$
V(P)=\oint_{C} V \bar{Q}(\Gamma) d s-\oint_{C} \frac{\partial V}{\partial n} \bar{M}(\Gamma) d s+\oint_{C} \bar{M}(V) \frac{\partial \Gamma}{\partial n} d s-\oint_{C} \bar{Q}(V) \Gamma d s+\int_{R} \Gamma \Delta^{2} V d x d y .
$$

For $P$ on the interior or $R$ we may differentiate (4.10) with respect to the variables of the point $P$ and interchange order of differentiation and integration on the right. Thus we obtain for $p \leqslant 3$,

$$
\begin{aligned}
V^{(p)}(O)=\oint_{C} V \bar{Q}\left(\Gamma^{(p)}\right) d s-\oint_{C} \frac{\partial V}{\partial n} \bar{M}\left(\Gamma^{(p)}\right) d s+\oint_{C} \bar{M}(V) \frac{\partial}{\partial n} \Gamma^{(p)} d s-\oint_{C} \bar{Q}(V) \Gamma^{(p)} d s & \\
& +\int_{R} \Gamma^{(p)} \Delta^{2} V d x d y .
\end{aligned}
$$

Here again as in the previous section we could add to $\Gamma^{(p)}$ any biharmonic function $B^{p}$. The last three terms of (4.11) involve only the known function $\Gamma$ and the data of $V$. Hence we define

$$
\Phi^{(p)}(O)=V^{(p)}(O)-\oint_{C} \bar{M}(V) \frac{\partial \Gamma^{(p)}}{\partial n} d s+\oint_{C} \bar{Q}(V) \Gamma^{(p)} d s-\int_{R} \Gamma^{(p)} \Delta^{2} V d x d y
$$

and obtain by Schwarz's inequality

$$
\left|\Phi^{(p)}(O)\right|^{2} \leqslant\left[\left(\frac{2 \pi}{L}\right)^{2} \oint_{C} V^{2} d s+\oint_{C}\left(\frac{\partial V}{\partial n}\right)^{2} d s\right]\left\{\left(\frac{L}{2 \pi}\right)^{2} \oint_{C}\left[\bar{Q}\left(\Gamma^{(p)}\right)\right]^{2} d s+\oint_{C}\left[\bar{M}\left(\Gamma^{(p)}\right)\right]^{2} d s\right\} .
$$

Since $\oint_{C} V d s=O$ it follows from (4.4) and (4.8) that

Thus

$$
\oint_{C} V^{2} d s \leqslant\left(\frac{L}{2 \pi}\right)^{2} \oint_{C}\left(\frac{\partial V}{\partial s}\right)^{2} d s
$$

$$
\left|\Phi^{(p)}(O)\right|^{2} \leqslant\left[\oint_{C}\left(\frac{\partial V}{\partial s}\right)^{2} d s+\oint_{C}\left(\frac{\partial V}{\partial n}\right)^{2} d s\right]\left\{\left(\frac{L}{2 \pi}\right)^{2} \oint_{C}\left[\bar{Q}\left(\Gamma^{(p)}\right)\right]^{2} d s+\oint_{C}\left[\bar{M}\left(\Gamma^{(p)}\right)\right]^{2} d s\right\} .
$$

Now

$$
\oint_{C}\left(\frac{\partial V}{\partial s}\right)^{2} d s+\oint_{C}\left(\frac{\partial V}{\partial n}\right)^{2} d s=\oint_{C}\left[\left(\frac{\partial V}{\partial x}\right)^{2}+\left(\frac{\partial V}{\partial y}\right)^{2}\right] d s
$$

In view of (4.3) and the fact that $\oint_{C}\left(\frac{\partial V}{\partial x}\right) d s=\oint_{C}\left(\frac{\partial V}{\partial y}\right) d s=O$ we have

$$
\oint_{C}\left[\left(\frac{\partial V}{\partial x}\right)^{2}+\left(\frac{\partial V}{\partial y}\right)^{2}\right] d s \leqslant \frac{1}{p_{2}} \int_{R}\left(V,{ }_{x i} V,{ }_{x i}+V,{ }_{y i} V,{ }_{y i}\right) d x d y=\frac{1}{p_{2}} \int_{R} V,{ }_{i j} V,{ }_{i j} d x d y .
$$

Thus from (4.14), (4.16), (4.17) and the definition of $E(V, V)$ we have for $\sigma>O$

$$
\left[\left(\frac{2 \pi}{L}\right)^{2} \oint_{C} V^{2} d s+\oint_{C}\left(\frac{\partial V}{\partial n}\right)^{2}\right] d s \leqslant \frac{2}{D p_{2}(1-\sigma)} E(V, V) .
$$

(For $-1 / 2<\sigma<O$ the factor $(1-\sigma)$ is replaced by $(1+2 \sigma)$.) From the divergence theorem we then obtain

$$
\left(\frac{2 \pi}{L}\right)^{2} \oint_{C} V^{2} d s+\oint_{C}\left(\frac{\partial V}{\partial n}\right)^{2} d s \leqslant \frac{2}{D p_{2}(1-\sigma)}\left\{\oint_{C} \frac{\partial V}{\partial n} \bar{M}(V) d s-\oint_{C} V \bar{Q}(V) d s\right\} .
$$


An application of Schwarz's inequality yields

$$
\left(\frac{2 \pi}{L}\right)^{2} \oint_{C} V^{2} d s+\oint_{C}\left(\frac{\partial V}{\partial n}\right)^{2} d s \leqslant\left[D \frac{2}{p_{2}(1-\sigma)}\right]^{2}\left\{\oint_{C}[\bar{M}(V)]^{2} d s+\left(\frac{L}{2 \pi}\right)^{2} \oint_{C}[\bar{Q}(V)]^{2} d s\right\}
$$

Insertion of (4.20) back into (4.13) then gives the desired bound

$$
\begin{aligned}
\left|\Phi^{(p)}(O)\right|^{2} \leqslant\left[\frac{2}{D p_{2}(1-\sigma)}\right]^{2}\left\{\oint_{C}[\bar{M}(V)]^{2} d s+\right. & \left.\left(\frac{L}{2 \pi}\right)^{2} \oint_{C}[\bar{Q}(V)]^{2} d s\right\} \\
& \left\{\oint_{C}\left[\bar{M}\left(\Gamma^{(p)}\right)\right]^{2} d s+\left(\frac{L}{2 \pi}\right)^{2} \oint_{C}\left[\bar{Q}\left(\Gamma^{(p)}\right)\right]^{2} d s\right\} .
\end{aligned}
$$

This is equivalent to the desired inequality (4.1), since instead of (4.12) we could have used Schwarz's inequality on the last three terms, being careful to use

$$
\int_{R} \Gamma^{(p)} \Delta^{2} V d x d y \leqslant \int_{R} r_{P P^{\prime}}\left[\Gamma^{(p)}\right]^{2} d x d y \int_{R} r_{P P^{\prime}}^{-1} \Delta^{2} V d x d y \text { for } p=3
$$

It remains now to derive a bound for $p_{2}$ (i.e., find a $\bar{p}_{2}$ ). We denote by $u_{2}$ the eigenfunction corresponding to $p_{2}$, i.e., (see (4.4) and (4.5))

in $R$ and

$$
\Delta u_{2}=O
$$

$$
\frac{\partial u_{2}}{\partial n}-p_{2} u_{2}=O
$$

on $C$. From (4.4) and (4.8) we have

$$
\oint_{C} u_{2}^{2} d s \leqslant\left(\frac{L}{2 \pi}\right)^{2} \oint_{C}\left(\frac{\partial u_{2}}{\partial s}\right)^{2} d s .
$$

We consider again the identity (see [5])

$$
\oint_{C} t\left(\frac{\partial u_{2}}{\partial s}\right)^{2} d s=\oint_{C} t\left(\frac{\partial u_{2}}{\partial n}\right)^{2} d s+2 \oint_{C} f^{i} \frac{\partial x^{i}}{\partial s} \frac{\partial u_{2}}{\partial n} \frac{\partial u_{2}}{\partial s} d s+\int_{R}\left[f,{ }_{k}, \delta_{i l}-2 f{ }_{l},{ }_{l}\right] u_{2, i} u_{2, l} d x d y
$$

which was used in deriving (3.34). Setting

$$
\int_{R}\left[f_{, k}^{k}, \delta_{i l}-2 f^{i},{ }_{l}\right] u_{2, i} u_{2, l} d x d y \leqslant c \int_{R} u_{2, i} u_{2, i} d x d y
$$

we obtain from (4.25) (by making use of the arithmetic-geometric mean inequality)

$$
t_{m} \oint_{C}\left(\frac{\partial u_{2}}{\partial s}\right)^{2} d s \leqslant \oint_{C} t\left(\frac{\partial u_{2}}{\partial s}\right)^{2} d s \leqslant \bar{b} \oint_{C}\left(\frac{\partial u_{2}}{\partial n}\right)^{2} d s+2 c \int_{R} u_{2, i} u_{2, i} d x d y
$$

where

$$
\bar{b}=2\left\{\left[2\left(f^{i} \frac{\partial x^{i}}{\partial s}\right)^{2}+t^{2}\right] / t\right\}_{\max } \leqslant 2\left\{2 b t_{m}^{-1}-t_{m}\right\}
$$

with $b$ given by (3.21). Inserting (4.28) into (4.24) and making use of (4.22) and (4.23) we obtain

$$
\oint_{C} u^{2} d s \leqslant\left(\frac{L}{2 \pi}\right)^{2} t_{m}^{-1}\left\{p_{2}^{2} \bar{b}+2 c p_{2}\right\} \oint_{C} u^{2} d s .
$$


It follows then that

$$
\left(p_{2}+\frac{c}{b}\right)^{2} \geqslant 4 \pi^{2} t_{m}\left(\bar{b} L^{2}\right)^{-1}+[c / \bar{b}]^{2}
$$

or that

$$
p_{2} \geqslant \bar{p}_{2} \equiv\left[4 \pi^{2} t_{m}\left(\bar{b} L^{2}\right)^{-1}+(c / \bar{b})^{2}\right]^{1 / 2}-c / \bar{b} .
$$

If the boundary $C$ of $R$ is star-shaped with respect to the origin (i.e., $x^{i} n_{i}>0$ ) then we may choose $f^{i}=x^{i}$, in which case $c=0, t=x^{i} n_{i}$, and $\bar{b}=2\left\{2 r^{2}{ }_{M} t_{m}^{-1}-t_{m}\right\}$.

Thus far we have indicated how to obtain bounds for the function and its first, second, and third derivatives. In physical problems this includes all derivatives for which we would normally desire bounds. However, if we wish bounds for higher derivatives they may be obtained using the techniques of the previous section. For interior bounds the only term which will cause difficulty for $p>3$ is the last term on the right of (4.12); but this is precisely the term which was evaluated in the previous section.

\section{References}

[1] Bramble, J. H. and Payne, L. E., Pointwise bounds in the first biharmonic boundary value problem, J. Math. Phys. (to appear).

[2] Bramble, J. H. and Payne, L. E., Bounds for derivatives in the Dirichlet problem for the Poisson equation, J. Soc. Ind. Appl. Math., 10, pp. $370-380$ (1962).

[3] Faber, C., Beweis, dass unter allen homogenen Membranen von gleicher Fläche und gleicher Spannung die kreisförmige den tiefsten Grundton gibt, Sitz. Bayer. Akad. Wiss. pp. 169-172 (1923).

[4] Krahn, E., Uber eine von Rayleigh formulierte Minimaleigenschaft des Kreises, Math. Ann., 94, pp. $97-100$ (1924).

[5] Payne, L. E. and Weinberger, H. F., New bounds in harmonic and biharmonic problems, J. Math. Phys 4, pp. 291-307 (1955).

(Paper 67B3-99) 